\title{
Dissecting causes for improved survival among patients with acute myeloid leukemia in two different eras receiving identical regimens in sequential randomized studies
}

\author{
Ing S. Tiong (1)', John Reynolds $\mathbb{B}^{2}$, Kenneth F. Bradstock ${ }^{3}$, John F. Seymour ${ }^{4}$ and Andrew H. Wei', on behalf of the \\ Australasian Leukaemia \& Lymphoma Group4
}

\begin{abstract}
Cytarabine and anthracyclines represent the core therapeutic drugs for acute myeloid leukemia (AML). Despite the paucity of therapeutic innovations, large populationbased registries have demonstrated incremental survival improvements over the decades, especially among younger patients ${ }^{1,2}$, potentially attributable to intensified chemotherapy, improved supportive care, or improved risk stratification and selection for allogeneic stem cell transplantation (SCT). However, it is difficult to ascertain the relative contribution of each to improvements in patient outcome.
\end{abstract}

The Australasian Leukemia and Lymphoma Group (ALLG) has conducted a series of randomized clinical trials in adult AML, leading to the stepwise incorporation of etoposide ${ }^{3}$ and high-dose cytarabine intensification in the induction phase ${ }^{4}$. Subsequently, both AMLM7 $(1995-2000)^{5}$ and AMLM12 (2003-2013) ${ }^{6}$ trials, spanning an 18-year treatment period, used an identical induction protocol (ICE: idarubicin $9 \mathrm{mg} / \mathrm{m}^{2}$ days $1-3$; cytarabine $3 \mathrm{~g} / \mathrm{m}^{2}$ twice a day on days $1,3,5$, 7; etoposide $75 \mathrm{mg} / \mathrm{m}^{2}$ days $\left.1-7\right)$ and shared a common consolidation control arm (IcE: idarubicin days 1-2; cytarabine $100 \mathrm{mg}$ / $\mathrm{m}^{2}$ days $1-5$; etoposide days $1-5 \times 2$ cycles) as part of a 1:1 randomization with an investigational regimen in the

\footnotetext{
Correspondence: Andrew H. Wei (andrew.wei@monash.edu)

'Department of Clinical Haematology, Alfred Hospital and Monash University, Melbourne, Australia

${ }^{2}$ Biostatistics Platform, Faculty of Medicine, Nursing and Health Sciences, Monash University, Melbourne, Australia
}

Full list of author information is available at the end of the article. post-remission phase. In AMLM7, the investigational arm included a second round of ICE, shown to be nonsuperior to standard IcE 5 . In AMLM12, anthracycline intensification incorporating an extra day of idarubicin in each of the two consolidation cycles was explored and shown to significantly improve leukemia-free survival ${ }^{6}$.

The overall survival (OS) in AMLM12 was superior to AMLM7 (median 44.3 vs. 24.8 months, $p=0.009$ ) (Fig. 1a). To explore reasons for the differences in OS, we compared between each study era: (1) early induction outcomes following the identical ICE induction regimen; (2) post-remission outcomes in patients receiving the common standard IcE consolidation arm; and (3) survival following disease relapse.

Cytogenetic risk was classified in both studies in accordance with the revised MRC classification ${ }^{7}$. Patients with favorable-risk karyotype were excluded from the AMLM7 cohort as this subgroup was excluded in AMLM12. OS was calculated from the start of treatment and relapse-free survival from the date of first remission (CR1). Kaplan-Meier survival curves were compared using log-rank statistics, hazard ratios by Cox proportional hazard model, and univariate/multivariate analyses by logistic regression model. All tests were two-sided and considered significant where $p<0.05$. $\mathrm{R}$ statistical software version 3.4.4 ( $\mathrm{R}$ foundation for statistical computing, Vienna, Austria) was used.

Table 1 summarizes patient and treatment characteristics. The AMLM7 cohort was younger (median 43 vs. 48 years, $p<0.001)$, less commonly received granulocyte 


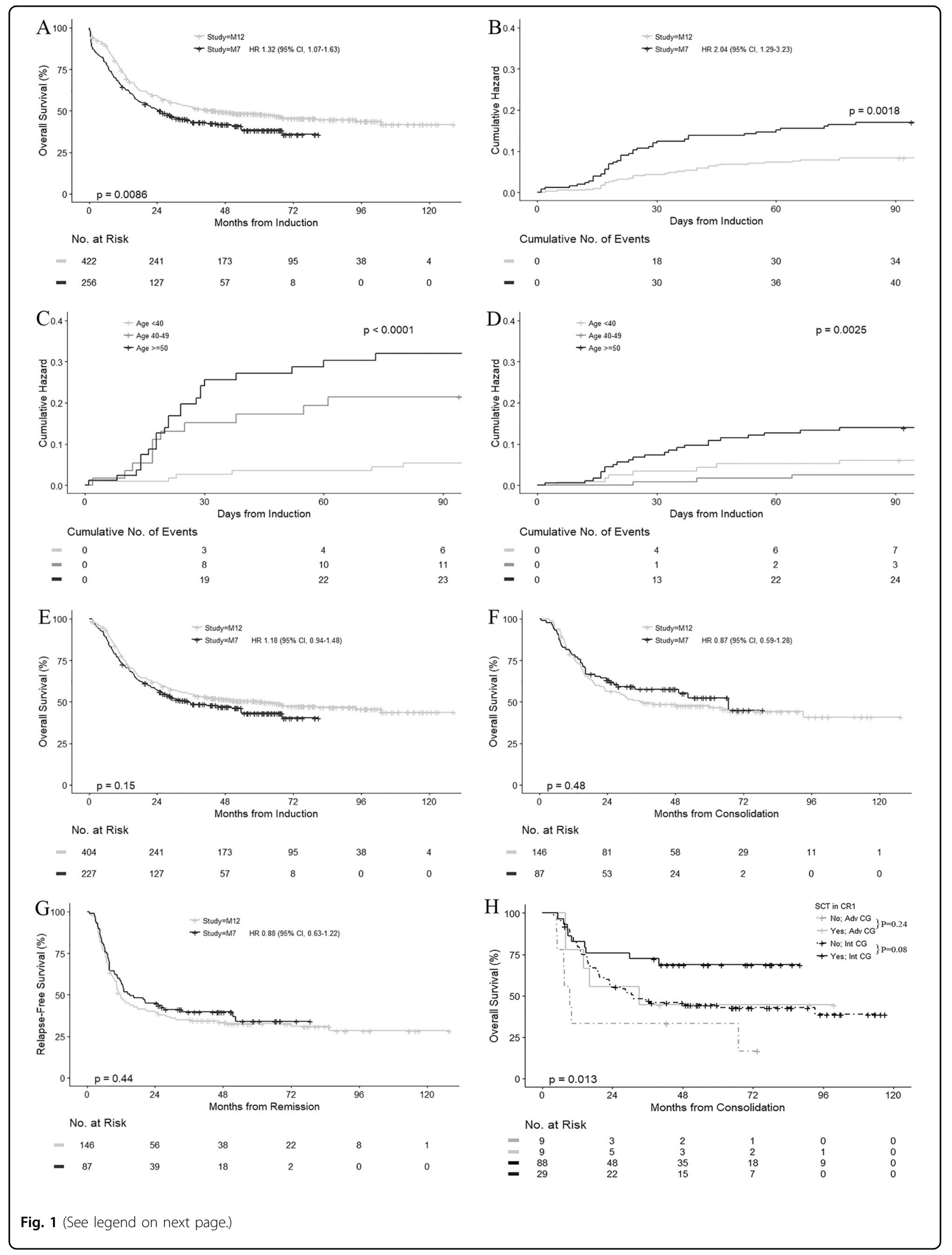


Fig. 1 Overall survival, relapse-free survival and cumulative hazard curves. a Overall survival comparing the AMLM7 and AMLM12 study cohorts. b Cumulative hazard of early deaths in the AMLM7 and AMLM12 study cohorts. c Cumulative hazard of early deaths in the AMLM7 cohort stratified by age groups. $\mathbf{d}$ Cumulative hazard of early deaths in the AMLM12 cohort stratified by age groups. e Overall survival by landmark analysis at 30 days in the AMLM7 and AMLM12 study cohorts. $\mathbf{f}$ Overall survival of patients receiving the common IcE consolidation chemotherapy. $\mathbf{g}$ Relapse-free survival in patients receiving the common IcE consolidation chemotherapy. $\mathbf{h}$ Overall survival in the AMLM12 study cohort receiving standard IcE consolidation chemotherapy, restricted to patients with $>90$ days of remission duration, stratified by allogeneic SCT in first remission and cytogenetic risk. Pairwise comparisons using log-rank test, with $p$-values adjusted by Benamini \& Hochberg (BH) method

colony-stimulating factor (G-CSF), less commonly received allogeneic SCT and more commonly received autologous SCT. Complete remission (CR) rates $(75 \%$ in AMLM7 and 76\% in AMLM12) were similar after the first cycle of ICE. Median follow-up duration was 51.5 and 71.5 months for survivors in AMLM7 and AMLM12, respectively.

We initially focused on early induction outcomes among 678 patients receiving the ICE induction protocol ( $n=256$ in AMLM7 and $n=422$ in AMLM12). Early deaths (at 30 days) were more common in AMLM7 (11.7\%) than AMLM12 (4.3\%) $(p=<0.001)$ (Fig. 1b), and strongly linked to increasing age: with higher mortality occurring in patients $>40$ years in AMLM7 (Fig. 1c) but only $>50$ years in AMLM12 (Fig. 1d). Although OS appeared improved in the AMLM12 study (Supplementary Figure S1A), this difference in OS was attenuated if OS was compared using a 30-day landmark analysis (Fig. 1e), confirming the initial divergence of the survival curves seen in Fig. 1a. Higher 30-day mortality (odds ratio 3.46, 95\% confidence interval, 1.83-6.69) was observed in the AMLM7 vs. the AMLM12 study eras (Supplementary Table S1). In addition to these inter-study differences, there also appeared a trend for reduced intra-study early deaths in the later half of the AMLM12 recruiting period (2007-2010), compared to the first half (2003-2006) (Supplementary Figure S1A-B).

The ICE induction protocol was identical except for the first 44 patients in the AMLM7 study who received idarubicin $12 \mathrm{mg} / \mathrm{m}^{2}$. Thirty-day mortality was not higher in this initial subgroup $(4 / 44 ; 9.1 \%)$. The remaining patients in AMLM7 and all patients in AMLM12 received the identical induction and consolidation regimen in the control arm. Hence, the reduction in early deaths in AMLM12 was not related to differences in chemotherapy intensity, and was likely attributable to improved supportive care practices in the AMLM12 study. Aspects of supportive care were explored where data were available (Supplementary Table S2).

Antifungal prophylaxis was documented in 75/87 (86\%) patients in AMLM7, all using fluconazole/itraconazole, and in 397/414 (96\%) patients in AMLM12, using fluconazole/itraconazole $(71 \%)$ or posaconazole/voriconazole (38\%). Many patients received $>1$ antifungal agent at different time points. AMLM12 patients who received mold-active antifungal prophylaxis had a trend for reduced incidence of documented fungal infections (4.7 vs. $10.2 \%, p=0.06)$, similar 30 -day mortality (3.4 vs. $3.8 \%$ ) and potentially lower 90 -day mortality ( 4.0 vs. $9.1 \%, p=$ 0.07 ), consistent with previously demonstrated benefits of posaconazole over fluconazole or itraconazole ${ }^{8}$. Strict definitions of possible/probable/definite invasive fungal infections were not available in the database and so could not be compared. The availability of mold-active antifungal agent in the later era may have contributed to the improved outcomes observed for patients recruited to the second half of the AMLM12 study era (Supplementary Figure S1C).

Although the use of G-CSF (80\% in AMLM7, 97\% in AMLM12) and palifermin (0 vs. $18 \%)$ were more frequent in the later AMLM12 cohort, published randomized trials did not demonstrate significant impact of either agent on induction deaths ${ }^{9,10}$. Around one-third of patients received prophylactic fluoroquinolones in both cohorts, with similar fever days and documented infections. Transfusion support was also similar for both red blood cells and platelets. The leading causes of death for both cohorts, where documented, were infection $(59 \%$ in AMLM7 and 39\% in AMLM12) and multiorgan failure (28\% in AMLM7 and 50\% in M12).

After examining early induction outcomes, we next compared post-remission outcomes in 233 patients $(n=$ 87 in AMLM7 and $n=146$ in AMLM12) randomized to the IcE consolidation control arm common to both studies. Despite older age and more patients with ECOG $>0$ in the AMLM12 cohort (Supplementary Table S3), there was no significant difference in either OS (median 36.4 months in AMLM12 vs. 66.7 months in AMLM7, $p=0.48$ ) (Fig. 1f) or relapse-free survival (median 11.8 months vs. 15.1 months, $p=0.44$ ) (Fig. 1g). Although significantly more patients underwent CR1 allogeneic SCT in AMLM12 (28 vs. 10\%), long-term post-remission survival outcomes were identical, despite matching for baseline characteristics, censoring for SCT, or treating SCT as a competing risk (Supplementary Figures S2-S3). Allogeneic SCT in CR1, however, was associated with improved survival in patients with intermediate $(n=117)$, but not adverse cytogenetic risk $(n=18)$ in the AMLM12 
Table 1 Patient characteristics and outcomes in the entire AMLM7 and AMLM12 cohorts

\begin{tabular}{|c|c|c|c|}
\hline & $\begin{array}{l}\text { AMLM7 }(n= \\
256)\end{array}$ & $\begin{array}{l}\text { AMLM12 }(n= \\
422)\end{array}$ & $p$-value ${ }^{a}$ \\
\hline Age, median years (IQR) & $43.4(31.6-51.6)$ & $48.1(38.4-55.2)$ & $<0.001$ \\
\hline Male gender (\%) & 55.1 & 57.1 & 0.6 \\
\hline ECOG PS > 0 (\%) & 50.0 & 54.9 & 0.2 \\
\hline \multicolumn{3}{|l|}{ Cytogenetics, n (\%) } & \multirow[t]{4}{*}{0.2} \\
\hline Intermediate & $201(78.5)$ & $338(80.1)$ & \\
\hline Adverse & $39(15.2)$ & $70(16.6)$ & \\
\hline Unknown & $16(6.3)$ & $14(3.3)$ & \\
\hline $\begin{array}{l}\text { WCC }\left(\times 10^{9} / L\right) \text {, median } \\
(I Q R)\end{array}$ & $10.6(3.3-43.5)$ & $10.0(2.9-33.7)$ & 0.3 \\
\hline$>40 \times 10^{9} / \mathrm{L}(\%)$ & 26.2 & 21.6 & 0.2 \\
\hline$>100 \times 10^{9} / L(\%)$ & 10.5 & 6.6 & 0.082 \\
\hline Febrile (\%) & 30.1 & 29.1 & 0.8 \\
\hline Bleeding (\%) & 22.3 & 12.8 & 0.002 \\
\hline DIC (\%) & 4.7 & 3.1 & 0.3 \\
\hline G-CSF (\%) & 80.1 & 96.7 & $<.001$ \\
\hline$>1$ induction cycle, $n(\%)$ & $19(7.4)$ & $41(9.7)$ & 0.3 \\
\hline CR-after 1 cycle (\%) & 75.4 & 76.3 & 0.8 \\
\hline CR-total (\%) & 79.7 & 82.5 & 0.4 \\
\hline $\begin{array}{l}\text { Randomized to standard } \\
I C E, n(\%)\end{array}$ & $87(34.0)$ & $146(34.6)$ & 0.9 \\
\hline $\begin{array}{l}\text { Received } 2 \text { cycles of } \\
\text { standard ICE }\end{array}$ & $81(93.1)$ & 125 (85.6) & 0.7 \\
\hline \multicolumn{4}{|l|}{ Early mortality, n (\%) } \\
\hline 30-day mortality & $30(11.7)$ & $18(4.3)$ & $<0.001$ \\
\hline 60-day mortality & $36(14.1)$ & $30(7.1)$ & 0.005 \\
\hline 90-day mortality & $40(15.6)$ & $34(8.1)$ & 0.003 \\
\hline Allogeneic SCT, n (\%) & $76(29.7)$ & $215(51.3)$ & $<0.001$ \\
\hline Transplant in first CR & $32(12.5)$ & $120(28.4)$ & $<0.001$ \\
\hline Transplant other status & $44(17.2)$ & $95(22.5)$ & 0.12 \\
\hline Autologous SCT, n (\%) & $25(9.8)$ & $7(1.7)$ & $<0.001$ \\
\hline
\end{tabular}

aFisher's exact test for categorical variables; Mann-Whitney $U$ tests for continuous variables

$C R$ complete remission, DIC disseminated intravascular coagulation, ECOG PS Eastern Cooperative Oncology Group performance status, G-CSF granulocyte colony stimulating factor, $I Q R$ interquartile range, SCT stem cell transplantation, WCC white cell counts

cohort (Fig. 1h). In AMLM7, SCT frequency was too low to enable meaningful interpretation of its impact (Supplementary Figure S4).

Among the entire AMLM7 and AMLM12 cohorts, 114 patients and 207 patients, respectively, experienced disease relapse associated with limited OS (median
6.5 months vs. 7.9 months) (Supplementary Figure S5A). Data on salvage therapy and outcome were not available, but more patients in AMLM12 (47\%) than AMLM7 (38\%) underwent subsequent allogeneic SCT $(p=0.10)$, where survival was significantly improved, compared to patients not transplanted (Supplementary Figure S5B).

The goal of high-dose cytarabine-based induction is the rapid achievement of high-quality $C R$ from the initial chemotherapy and reduce the likelihood of re-induction therapy, sparing patients the risk of additional complications. ICE results in a very high first-cycle CR rate $(\sim 76 \%$ excluding favorable-risk AML), compared to 59 and $71 \%$ after 1 and 2 cycles of $7+3\left(90 \mathrm{mg} / \mathrm{m}^{2} \text { daunorubicin }\right)^{11}$. Our analysis of randomized clinical trials from two different eras demonstrates that ICE induction has become more tolerable over time, especially in those $<50$ years, likely from multi-faceted improvements in supportive care. Interestingly, a volume effect was evident in the AMLM12 (but not in the AMLM7 study), where patients in the top five recruiting centers had better survival outcome (Supplementary Figure S6); similar effects have been observed by others ${ }^{12}$. Early deaths from ICE induction in AMLM12 were 2.1 and $7.0 \%$ in patients $<50$ and $\geq 50$ years. This compares favorably to other AML studies conducted in a similar era: $5.5 \%$ induction deaths in an ECOG study (2002-2008) ${ }^{11}$, and 5.5 and $10.1 \%$ in patients $<46$ and $\geq 46$ years in the EORTC-GIMEMA AML12 study (1999-2008) ${ }^{13}$.

Although a crude comparison between the AMLM12 and AMLM7 studies indicated a significant improvement in OS, potentially attributable to chemotherapy intensification in CR1, our analyses demonstrate that a substantial component of this benefit may also be linked to nonchemotherapy related factors. By examining deaths occurring during the induction and consolidation phases separately, we find that the major effect on survival was related to reduced treatment-related mortality during the induction phase of AMLM12 associated with improvements in supportive care practices, although the precise factors could not be fully determined. In the postremission setting, there was no major difference in relapse-free or OS, despite the increased incidence of allogeneic SCT in the more recent AMLM12 study. In conclusion, these findings demonstrate the complexities in making interpretations between identical treatment regimens delivered in sequential eras and highlights the importance of ensuring major practice changing decisions are based on prospectively conducted randomized controlled trials.

\section{Acknowledgements}

Contributors to the Australasian Leukemia \& Lymphoma Group AMLM12 trial Royal Melbourne Hospital: J. Szer, A. Grigg, A. Roberts; Westmead Hospital: W. Benson, M. Hertzberg, J. Koutts, K. Bradstock, A. Johnston, C. Turtle, I. Kerridge, F. Kwok; Calvary Mater Newcastle: A. Enno, P. Rowlings, S. Deveridge, M. Seldon, 
A. Enjeti, M Walsh, M. Seldon; Princess Alexandra Hospital: P. Marlton, A. Mills, D. Gill, P. Mollee, P. Wood, M. Gandhi, H. Middleton, S. Mapp, S. Hanlon, R. Bird, A. Henden, J. Seymour, M. Wolf, M. Prince, H. Januszewicz, D. Ritchie, W. Rasheed, S. Harrison; Box Hill Hospital: A. Schwarer, A. Wei, J. McKendrick; Royal Adelaide Hospital: I. Lewis, P. Bardy, P. Giri, N. Horvath, C-H. Hui, N. Patton, F. Szabo, B. To; Canberra Hospital: A. McDonald, I. Prosser, J. D'rozario, M. Pidcock, P. Crispin, D. Tallaulikar; Royal North Shore Hospital, C. Arthur, C. Ward, L. Coyle, K. Fay, N. Mackinley, M. Greenwood, S. Mulligan, W. Stevenson; Sir Charles Gairdner Hospital: G. Cull, D. Joske, D. Auguston, S. Ward, P. Crawford; Geelong Hospital: P. Campbell, R. Bell, R. McLennan; Alfred Hospital, A. Wei, A. Spencer, S. Avery, S. Patil; Fremantle Hospital: A. McQuillan, M. Leahy, F. Cordingley, J. Cooney, T. Calogero; Queen Elizabeth Hospital, U. Hahn, W. Jaksic, M. Kee, P. Bardy, S. Mcrae; Royal Perth Hospital, P. Cannell, B. Carnley, J. Cooney; Gosford Hospital: C. Tiley, M. Dean, B. Wylie; Royal Hobart Hospital: R. Harrup, R. Kimber, J. Daley, R. Lowenthal; St Vincent's Hospital Sydney, K. Fay, S. Milliken, J. Moore, D. Yeung, T. Dodds; Woollongong Hospital: K. Cartwright, P. Presgrave, P. Warburton; Concord Hospital, J. Trottman, L. Y. Kwan; Nepean Hospital: J. Taper, S. Fuller; Mater Brisbane Hospital: L. Catley, M. Ashish; Townsville Hospital: I. Irving. Contributors to the Australasian Leukemia \& Lymphoma Group AMLM7 trial-Westmead Hospital: J. Koutts, W. Benson, K. Bradstock, D. Gottlieb, M. Hertzberg, P. Castaldi; Royal Prince Alfred Hospital: D. Joshua, H. Kronenberg, J. Gibson, G. Young, H. Iland; Wesley Medical Center: P. Eliadis, J. Bashford, I. Bunce, S. Fanning, J. Morton; Calvary Mater Newcastle, S. Deveridge, A. Enno, M. Seldon, A. Spencer, I. Kerridge; Royal Brisbane Hospital, S. Durrant, A. Gillett, A. Nicol, I. Bunce; Princess Alexandra Hospital: D. Gill, P. Marlton, G. Cull; Liverpool Hospital: D. Rosenfeld, P. Motum, J. Gallo, I. Dunlop; Monash Medical Center: J. Catalano, E. Gan; P. Cannell, R. Herrmann, R. Baker; St George Hospital: T. Brighton, L. Y. Kwan; Royal North Shore Hospital: C. Arthur, D. Ma, K. Fay, R. McKinley, C. Ward; Alfred Hospital: A. Schwarer, P. Elliott, A. Spencer; Mater Brisbane Hospital: K. Taylor, S. Wright; Royal Melbourne Hospital, J. Szer, A. Grigg, P. Bardy, A. Roberts; Fremantle Hospital: F. Cordingley, M. Leahy, M. Webb; Royal Hobart Hospital: R. Lowenthal, R. Kimber, D. Jupe; Royal Adelaide Hospital: N. Horvath, J. Ho, T. Hughes, J. Dart; Peter MacCallum Cancer Center: H. Januszewicz, M. Wolf; Sir Charles Gairdner Hospital: S. Rule, D. Joske; Canberra Hospital: I. Prosser, M. Webb, I. Pidcock; Medical Oncology Clinic of Rosebank, Johannesburg, South Africa: B. Rapoport; Pretoria Academic Hospital, Pretoria, South Africa: C. Falkson; Austin Hospital: C. Smith; Repatriation Hospital Concord: I. Cunningham; Prince of Wales Hospital: R. Lindeman, P. Rowlings; Queen Elizabeth Hospital: J. Norman. Hematology Society of Australia and New Zealand Young Investigator Award for IST.

\section{Author details}

'Department of Clinical Haematology, Alfred Hospital and Monash University, Melbourne, Australia. ${ }^{2}$ Biostatistics Platform, Faculty of Medicine, Nursing and Health Sciences, Monash University, Melbourne, Australia. ${ }^{3}$ Department of Haematology, Westmead Hospital and University of Sydney, Sydney, Australia. ${ }^{4}$ Integrated Department of Haematology, Peter MacCallum Cancer Centre and Royal Melbourne Hospital, Melbourne, Australia

\section{Conflict of interest}

The authors declare that they have no conflict of interest.

\section{Publisher's note}

Springer Nature remains neutral with regard to jurisdictional claims in published maps and institutional affiliations.

Supplementary Information accompanies this paper at (https://doi.org/ 10.1038/s41408-018-0121-4).

Received: 7 June 2018 Revised: 21 July 2018 Accepted: 1 August 2018 Published online: 22 August 2018

\section{References}

1. Derolf A. R., et al. Improved patient survival for acute myeloid leukemia: a population-based study of 9729 patients diagnosed in Sweden between 1973 and 2005. Blood 2009, 113, 3666-3672.

2. Pulte, D., Gondos, A. \& Brenner, H. Improvements in survival of adults diagnosed with acute myeloblastic leukemia in the early 21 st century. Haematologica 93, 594 (2008).

3. Bishop, J. F. et al. Etoposide in acute nonlymphocytic leukemia. Australian Leukemia Study Group. Blood 75, 27-32 (1990).

4. Bishop, J. F. et al. A randomized study of high-dose cytarabine in induction in acute myeloid leukemia. Blood 87, 1710-1717 (1996).

5. Bradstock, K. F. et al. A randomized trial of high-versus conventional-dose cytarabine in consolidation chemotherapy for adult de novo acute myeloid leukemia in first remission after induction therapy containing high-dose cytarabine. Blood 105, 481-488 (2005).

6. Bradstock, K. F. et al. Idarubicin dose escalation during consolidation therapy for adult acute myeloid leukemia. J. Clin. Oncol. 35, 1678-1685 (2017).

7. Grimwade, D. et al. Refinement of cytogenetic classification in acute myeloid leukemia: determination of prognostic significance of rare recurring chromosomal abnormalities among 5876 younger adult patients treated in the United Kingdom Medical Research Council trials. Blood 116, 354-365 (2010).

8. Cornely, O. A. et al. Posaconazole vs. fluconazole or itraconazole prophylaxis in patients with neutropenia. N. Engl. J. Med. 356, 348-359 (2007).

9. Bradstock, K. et al. Effects of glycosylated recombinant human granulocyte colony-stimulating factor after high-dose cytarabine-based induction chemotherapy for adult acute myeloid leukaemia. Leukemia 15, 1331-1338 (2001).

10. Bradstock, K. F. et al. A randomized trial of prophylactic palifermin on gastrointestinal toxicity after intensive induction therapy for acute myeloid leukaemia. Br. J. Haematol. 167, 618-625 (2014).

11. Fernandez, H. F. et al. Anthracycline dose intensification in acute myeloid leukemia. N. Engl. J. Med. 361, 1249-1259 (2009).

12. Giri, S. et al. Impact of hospital volume on outcomes of patients undergoing chemotherapy for acute myeloid leukemia: a matched cohort study. Blood 125, 3359-3360 (2015).

13. Willemze, R. et al. High-dose cytarabine in induction treatment improves the outcome of adult patients younger than age 46 years with acute myeloid leukemia: results of the EORTC-GIMEMA AML-12 trial. J. Clin. Oncol. 32, 219-228 (2014). 